Aim To keep mothers and babies together and decrease term hypoglycaemia admissions through implementation of a new guideline for management of healthy term babies with asymptomatic hypoglycaemia in the first 48 hours; in line with the British Association of Perinatal Medicine neonatal hypoglycaemia framework for practice, by the 1st of October 2018.

Methods Hypoglycaemia threshold in healthy term babies $<48$ hours was redefined as $\leq 2.0 \mathrm{mmol} / \mathrm{l}$ (previously $\leq 2.6 \mathrm{mmol} / \mathrm{l}$ ) and the use of buccal dextrose gel in its treatment.

A multidisciplinary group was set up in the level 3 NNU. The guideline was disseminated using a 'read and sign' approach. A buccal dextrose monograph was developed. The blood gas analyser on labour ward was upgraded to allow neonatal sampling. Champions supported implementation through educational 'buzz' sessions and training videos. Test PDSAs were carried out, resulting in further training and guideline revision.

Results Baseline data revealed that hypoglycaemia made up $4 \%$ of term admissions (2\% full admissions, $2 \%$ short stay $\leq 4$ hours $\{S S\}) .37 \%$ of babies required IV fluids, $44 \%$ required nasogastric feeds and $19 \%$ were given oral formula top-up feeds. $41 \%$ of women intended to exclusively breastfeed, $7 \%$ were doing so at discharge. For quality assurance, a prospective audit of blood gas glucose and lab blood glucose was carried out. Our outcome measure is $\%$ of term admissions to NNU with hypoglycaemia. Since implementation there is a trend towards reduction in admissions - 2 SS admissions only. Our process measure is compliance with the hypoglycaemia bundle - currently 65\%. Our balance measures are: any adverse events, incorrect protocol (term vs preterm) use and an increase in breastfeeding rates. 1 infant $>48$ hours with no hypoglycaemia risk factors was treated on this protocol. There has been no incorrect protocol use. $72 \%$ of women intended to breastfeed, $47 \%$ were exclusively breastfed at discharge.

Conclusion There is a trend towards reduction in separation of mothers and babies. Bundle compliance requires ongoing work. Understanding barriers has been key to maintaining interdisciplinary staff engagement. There are ongoing challenges around equipment, the use of two different thresholds in term and preterm infants and avoiding person dependency.

\section{G82 CHANGING THE 'NO CHANGE' CULTURE: INCREASING USEFULNESS OF THE SCBU WARD ROUND}

${ }^{1} \mathrm{HF}$ Marshall, ${ }^{2} \mathrm{R}$ Ajitsaria, ${ }^{1} \mathrm{AM}$ Taylor, ${ }^{1} \mathrm{CE}$ Strauss, ${ }^{3} \mathrm{~N}$ Davey. ${ }^{1}$ Paediatric Department, North Middlesex University Hospital, London, UK; ${ }^{2}$ Paediatric Department, Hillingdon Hospital, London, UK; ${ }^{3}$ Quality Improvement Clinic, London, UK

\subsection{6/archdischild-2020-rcpch.63}

Aim This Quality Improvement project aimed to increase the usefulness of ward rounds in a busy Special Care Baby Unit (SCBU; i.e. low dependency neonatal unit). Most babies' primary reasons for remaining inpatient in SCBU are to gain weight and establish oral feeds. Despite this taking weeks for some, they were seen daily on ward round. Baseline data showed that $86 \%$ ward round plans for these low risk babies made no changes to management. My aim was to halve this number in four months.

Method Babies that required daily medical review (table 1) were identified and excluded from the project, leaving only low risk infants. The medical team were instructed to actively seek out babies of concern by discussion with nurses before ward round.

\section{Abstract G82 Table 1}

Exclusions

-Any nursing concerns (ask explicitly)

-Any parental concerns

- On treatment dose antibiotics, intravenous fluids, morphine

-Being observed for drug withdrawal

-Known cardiac defect that could lead to heart failure

-'Rooming $\ln ^{\prime}$ prior to discharge

The first PDSA cycle introduced the project to colleagues. Subsequent cycles reduced the frequency of ward round to three times per week, trialling different days. Data was collected from clinical notes. Balancing measures were details of babies that became unwell in the data collection period.

Results The frequency of ward round plans making no changes to management of low risk babies dropped from $86 \%$ to $17 \%$, which surpassed the aim. Doctors felt they had more time to help colleagues on the postnatal ward, complete discharge paperwork further in advance (thus facilitating morning discharges) and for projects. Medical and nursing teams were satisfied that well babies were not examined and disturbed daily.

Conclusion Medical and nursing teams are more satisfied with ward rounds occurring three times per week instead of daily for low risk babies in SCBU. More work is needed to look into ways to empower nursing staff to lead on care in low risk babies on their journey to discharge. This project would have had more impact if a reliable measure could be found for staff efficiency.

\section{G83 REDUCING LENGTH OF STAY FOR CHILDREN WITH PROLONGED ADMISSIONS TO HOSPITAL}

${ }^{1,2} \mathrm{M}$ Butler, ${ }^{1,2} \mathrm{~J}$ Gough. 'General Paediatrics, Evelina London, London, UK; '2Service Improvement Team, Evelina London, London, UK

\subsection{6/archdischild-2020-rcpch.64}

Aims To reduce the length of stay for patients with prolonged admission to a tertiary paediatric hospital. To improve experience for patients by reducing the disruption to their personal and family life, development and education, reduce discomfort and risk of healthcare associated harm. To improve capacity for the institution to undertake additional activity, improving financial performance. This additional activity would also reduce waiting times and improve access to care for other children. To improve the experience for health care professionals by enabling better care and reducing conflict.

Methods We systematically collected data on length of stay for inpatients across our institution (excluding neonatology) retrospectively and prospectively. We reviewed this data and shared it with clinicians (and continue to do so), we engaged with families and staff to identify their priorities and learn from their experiences. We initiated a range of projects related to this theme, including new care standards, new electronic documentation, education and collaboration sessions, new working practices and new staff roles. We developed a governance structure to monitor and sustain these new approaches, and are continuing to work on continuous improvement across a range of metrics. 
Results Our primary measure 'bed days per month occupied by patients with a length of stay of over 30 days' decreased from a mean of 635 bed days/month in 2016-18 to 534 bed days/month in $2018 / 19$, a reduction of $16 \%$. This is equivalent to $>3$ additional beds available on the wards each day.

In 2019 year to date we have sustained this reduction and aim for further improvement. In addition, patient and staff satisfaction with the care of these patients has improved and the rate of readmission was unaffected.

Conclusion Despite increasing medical complexity in our inpatients, and internal and external challenges the rise we had seen in prolonged admissions over recent years is not inevitable. It is possible to significantly reduce length of stay for these patients and simultaneously improve quality of care. Improving services for these children and young people should be a priority for paediatrics in the future and has benefits for patients, families, staff, the institution and the wider population.

\section{G84 IMPROVING BCG VACCINATION REFERRAL OF ADMITTED ELIGIBLE NEWBORN TO 98\% BY JULY 2019}

KJ Jeyasingam, HM McElroy. Neonatology, Medway Maritime Hospital, Medway, UK

\subsection{6/archdischild-2020-rcpch.65}

Aim The development of effective antibiotics and the BCG vaccination has significantly reduced the number of TB cases. The aim of this quality improvement project (QIP) is to improve BCG vaccination referrals for admitted eligible newborns in Hospital to $98 \%$ by June-July 2019, by raising awareness amongst healthcare professionals and improving identification of eligible newborns.

Methodology The aim and objectives were formulated using a driver diagram. A Plan-Do-Study-Act cycle was used to identify suitable changes and outcomes. Euroking database was used to create a spreadsheet with information specific for BCG vaccination eligibility and referral status. Data was collected from May 2018 to May 2019. Resources that raised awareness amongst healthcare professionals, by providing guidance for easier recognition of eligible newborns, were created by working closely with doctors, nurses and midwives.

Result The numbers of missed BCG referrals from May 2018 to Aug 2018 was 10 out of 13 (76. 9\%). From September 2018 to May 2019, it was 18 out of 34 (52.9\%), which showed a reduction of $23.9 \%$ in missed BCG referrals. From September 2018 onwards, doctors were obliged to refer newborns for BCG vaccination.

Questionnaires were sent out in March 2019 to former and current paediatric and non-paediatric doctors. 7 out of 10 questionnaire responses were received. All of the responders found it easy to refer newborns for BCG vaccination and used the WHO list to identify high-risk countries. Paediatric trainees were informed about BCG vaccination referral during their induction, whilst non- paediatric trainees were aware of the referral via this QIP or by speaking to seniors. In May 2019, awareness was raised and random spot checks were done in June and July 2019.The number of missed BCG referrals for June 2019 to July 2019 was 0 out of 2 and $100 \%$ of BCG referrals was achieved.

Conclusion Improving BCG referrals will reduce the time, work and resources needed to chase missed BCG referrals and avoid new cases of TB. This could be achieved with easy access to information, such as the ethnic origins of grandparents/parents of newborns, which will be added to the next antenatal book.

\section{G85 IMPROVING CANNULATION EFFICIENCY IN LEEDS CHILDREN'S HOSPITAL (LCH)}

A Fonfe, K Lotha, T Rance. Paediatrics, Leeds Teaching Hospitals, Leeds, UK

\subsection{6/archdischild-2020-rcpch.66}

Background There was anecdotal evidence that the time to gather equipment for paediatric cannulation was time consuming. This had the potential to create delays in such time-crucial matters as IV antibiotics within 60 minutes in suspected sepsis.

Aim To standardise cannulation trolleys across LCH to improve equipment gathering efficiency.

Method

- Questionnaire to junior doctors to determine how long it equipment gathering takes and number of cannulas inserted per shift.

- Junior doctors were timed gathering all necessary cannulation equipment on all wards

- A pilot cannulation trolley was trialled on the children's assessment unit. Adjustments were made after feedback and photographs of equipment were inserted on the trolley to faciliate appropriate stocking.

- Junior doctors were then timed gathering cannulation equipment with the new trolleys.

- A cannulation trolley champion was nominated for each ward.

- New cannulation trolleys were disseminated across all wards in $\mathrm{LCH}$.

Results

- $88 \%$ of doctors agreed that 'It often takes me longer to find the equipment I need for cannulation than inserted the cannula'.

- 1-6 cannulas are inserted per junior doctor shift.

- Time taken to gather cannulation equipment at baseline 4.21 minutes. With cannulation trolleys 2.12 minutes.

- Based on 1-6 cannulations per doctor per shift, before the trolleys this would take $87-522$ minutes and post-trolleys 43-258 minutes.

Conclusion Introduction of standardised cannulation trolleys has improved efficiency at cannulation preparation and has enabled speedier cannulation especially in time critical circumstances such as sepsis.

\section{G86 IMPROVING DISCHARGE SUMMARY QUALITY IN A GREENFIELD SINGLE ROOM QUATERNARY NEONATAL INTENSIVE CARE UNIT}

${ }^{1,2} \mathrm{~A}$ Anbu Chakkarapani, 'L Chicoine, ${ }^{1} \mathrm{R}$ Soni, ${ }^{1} \mathrm{E}$ Elfaki A Alla, ${ }^{1} \mathrm{~J}$ Powell, ${ }^{1} \mathrm{~B}$ George, ${ }^{1} \mathrm{~L}$ Greig, ${ }^{1,2} \mathrm{~B}$ Lee. 'Division of Neonatology, Department of Pediatrics, Sidra Medicine, Doha, Qatar; ${ }^{2}$ Department of Pediatrics, Weill Cornell Medicine-Q, Doha, Qatar

\subsection{6/archdischild-2020-rcpch.67}

Background Neonatal Intensive Care Unit (NICU) Discharge Summary (DS) provides continuity of care and ensures safe patient transition between hospital and community. Inadequate NICU DS documentation contributes to medical errors and loss of follow up. Our goal is to improve the DS quality 